resistance and a lack of predictive biomarkers means that matching the best treatment options to patients is difficult. This study aims to characterise the extent of spatial and temporal ITH in advanced stage HGSOC at presentation and relapse.

Methodology Patients $(n=49)$ having maximal effort upfrontdebulking surgery for advanced HGSOC underwent a tumour mapping of their tumour dissemination patterns. Tumour biopsies were collected (range 4-15, median 9), when relapsed patients also had paired biopsies collected for genomic and phenotypic analysis. DNA was extracted from tumours (5 per patient, $n=49$ patients plus relapse samples) and Illumina Human OmniExpress genotyping performed. Allele-specific copy number $(\mathrm{CN})$ was quantified using ASCAT. Genomic heterogeneity was quantified as the estimated number of $\mathrm{CN}$ aberration events distinct between each pair of tumour deposits. Clonal diversity within a patient's deposits was calculated using the difference between within-patient and betweenpatient heterogeneity. Ki67 proliferation index was assessed from tumour sections collected prior to DNA extraction.

Result(s)* Extensive genomic variations in patterns of evolution for different patients' tumours was observed, including the relationship between matched primary tumours and relapsed disease. Widespread variations in CCNE1, MYC and PTEN CN were observed across multiple disseminated tumours in the same patients, and higher CCNE1 correlated with poor patient outcome $(p=0.038)$. Extensive heterogeneity in Ki67 proliferation index was observed across the cohort, $77 \%$ of patients had tumour scores covering low, moderate and/or high Ki67 scoring categories.

Conclusion* Broad ITH was observed at the genomic level across this cohort. Extensive $\mathrm{CN}$ variations in genes such as CCNE1, across multiple disseminated samples within patients, and widespread variations in proliferative index between multi-site tumours, indicates that a single tumour biopsy does not accurately depict disseminated HGSOC biology, and therefore should not be used for as a basis for prediction of patient prognosis or outcomes.

\section{RADIOMICS AND MOLECULAR CLASSIFICATION IN ENDOMETRIAL CANCER (THE ROME STUDY): A STEP FORWARD TO A SIMPLIFIED PRECISION MEDICINE}

${ }^{1} \mathrm{G}$ Bogani*, ${ }^{2} \mathrm{C}$ Castiglioni, ${ }^{1} \mathrm{~V}$ Chiappa, ${ }^{1} \mathrm{~F}$ Raspagliesi*. ' Istituto Nazionale dei Tumori | Fondazione IRCCS, Milano, Italy; ${ }^{2}$ Building U6 - University of Milano-Bicocca, Milano, Italy

\subsection{6/ijgc-2021-ESGO.591}

Introduction/Background* Molecular/genomic profiling is the most accurate method to assess prognosis of endometrial cancer patients. Similarly, the adoption of radiomics showed important results for screening, diagnosis and prognosis, across various radiological systems and oncologic specialties. Here, we aim to correlate radiomic features obtained from ultrasound images with the molecular/genomic profiling to identify new hallmarks for stratification of endometrial cancer patients into different classes of risk.

Methodology

This prospective single-arm observational study Patients with newly diagnosed endometrial cancer will have ultrasonographic evaluation and radiomic analysis of the ultrasonographic images. Then patients will have surgery followed by molecular/genomic evaluation. We will correlate radiomic features with molecular/genomic profiling to classify prognosis. Major Inclusion/Exclusion Criteria : Consecutive patients (aged 18 years or more) with newly diagnosed endometrial cancer. Patients should have preoperative ultrasonographic evaluation followed by surgery.

Result(s)* The central hypothesis is that combining radiomic features with molecular features might allow identifying various classes of risk for endometrial cancer, e.g. predicting unfavorable molecular/genomic profiling. The rationale for the proposed research is that once validated, radiomics applied to ultrasonographic images would be an effective, innovative and cheap method for tailor operative and postoperative treatment modality in endometrial cancer. Primary Endpoint : The main endpoints will: (i) to define the mechanism by which radiomic features predict the classification of endometrial cancer into various classes of risk e.g. predicting unfavorable molecular/ genomic profiling as defined by molecular classification; (ii) to determinate the scaled impact of radiomic features assessed on ultrasonographic images of endometrial tumors; and (iii) to assess the intraobserver and interobserver reproducibility of radiomic features on ultrasonographic images of endometrial tumors. Overall, 100 patients for study cohort and 40 for the validation cohort.

Conclusion* We expect that the radiomic analysis of ultrasonographic images by means of radiomic classifier of risks will provide comparable results to molecular/genomic. (Trial Registration: GR-2019-12370566 Bando per la Ricerca Finalizzata 2019, Ministero della 24 Salute, Repubblica Italiana)

\section{RAD51 RING TRIAL: A EUROPEAN INTERLABORATORY ANALYTICAL VALIDATION TO DETERMINE THE ROBUSTNESS OF RAD51 AS A BIOMARKER FOR HOMOLOGOUS RECOMBINATION}

${ }^{1} \mathrm{C}$ Kramer*, ${ }^{2} \mathrm{~A}$ Llop-Guevara, ${ }^{3} \mathrm{E}$ Yaniz-Galende, ${ }^{4} \mathrm{~B}$ Pellegrino, ${ }^{1} \mathrm{~N}$ Ter Haar, ${ }^{4} \mathrm{~N}$ Campanini, ${ }^{4} \mathrm{~A}$ Musolino, ${ }^{3} \mathrm{~A}$ Leary, ${ }^{5} \mathrm{M}$ Vreeswijk, ${ }^{2} \mathrm{~V}$ Serra, ${ }^{1} \mathrm{~T}$ Bosse. ${ }^{1}$ Leiden University Medical Center (LUMC), Pathology, Leiden, Netherlands; '2VHIO Vall d'Hebron Institut d'Oncologia, Experimental Therapeutics Group, Barcelona, Spain; ${ }^{3}$ Hospital Gustave Roussy, Oncological Medicine, Villejuif, France; ${ }^{4}$ University Hospital of Parma, Medical Oncology and Breast Unit, Medicine , Parma, Italy; ${ }^{5}$ Leiden University Medical Center (LUMC), Human Genetics, Leiden, Netherlands

\subsection{6/ijgc-2021-ESG0.592}

Introduction/Background* RAD51 protein has been proposed as a functional readout of homologous recombination (HR) status using formalin-fixed paraffin embedded (FFPE) tumour tissue blocks. Recently, few laboratories have assessed the performance of RAD51 as a predictive biomarker. However, the robustness of the test when applied in different laboratories has not been systematically investigated so far. In this study, we performed an interlaboratory $(n=4)$ analytical validation to determine the interobserver variability and the effect of (subtle) differences in the co-immunofluorescence (co-IF) protocol and microscope technicalities on RAD51 scores.

Methodology The RING trial cohort comprised of 12 highgrade serous ovarian cancer cases. On unstained serial sections of FFPE tumour tissue blocks, a co-IF staining with RAD51 and geminin was performed: (1) centrally in Vall d'Hebron Institute of Oncology and (2) locally in participating centers. The centrally stained slides were distributed among 


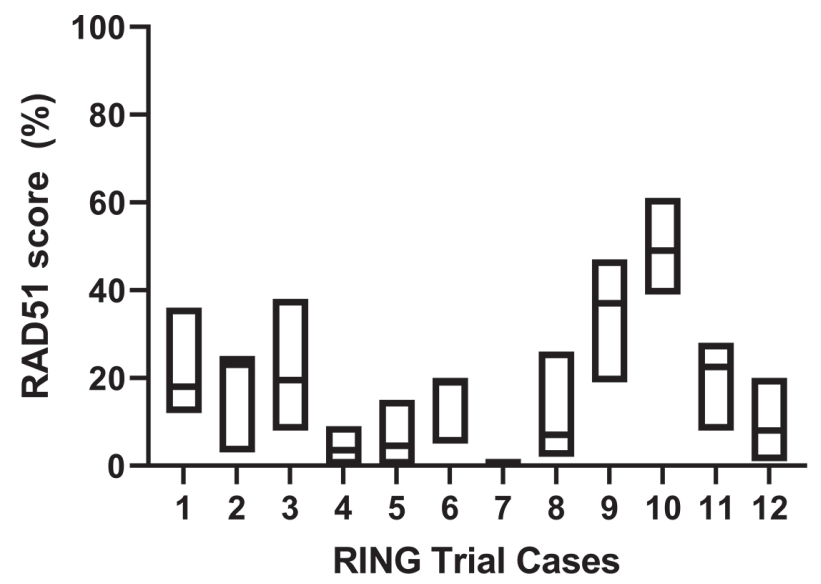

Abstract 376 Figure 1 Violin plot showing the varaiability in RAD51 scores (foci cut-off: $n=3$ ) between four observers. RAD51 scores were based on centrally stained slides. Lines in the plot indicate the median, min and max and RAD51 scores

participating centers for local RAD51 scoring. For the scope of the RING trial, a predefined and uniform scoring methodology was applied. Scoring was performed blinded for genetic and clinical data. Specific features in the analysis of the co-IF, including the number of RAD51 foci per nucleus and the presence of RAD51 subclonality, i.e., distinct RAD51 positive and negative areas, were incorporated in the RAD51 scoring form. For non-normally distributed data, variability was analyzed using the median, $25^{\text {th }}$ percentile $(\mathrm{Q} 1)$ and $75^{\text {th }}$ percentile (Q3).

Result(s)* Median variability in RAD51 scores between observers in centrally stained slides was 21\% (Q1: 15\%; Q3: 24\%) (figure 1). For the majority of cases $(n=10 / 12)$, a limited interobserver variability, defined as $\geq 3$ observers with scores in a narrow range, was detected. In contrast, in cases where observers noted granular pannuclear RAD51 staining or RAD51 background, there was a substantial variability in scores (figure 1; case 6 and 8). Median variability in RAD51 scores between centrally and locally stained IF slides was 7.7\% (Q1: 4.1\%; Q3: 11.7\%).

Conclusion* This is the first cross-European interlaboratory assessment of the performance of RAD51/Geminin co-IF. We show that subtle local protocol differences do not impact final RAD51 scores. Furthermore, we elucidated features that may negatively impact RAD51 score accuracy.

\section{EFFICACY ANALYSIS OF NIRAPARIB USING PATIENT- DERIVED XENOGRAFT OF RARE SUBTYPES OF OVARIAN CANCER}

${ }^{1} \mathrm{H}$ Asano*, ${ }^{2,3} \mathrm{KC}$ Hatanaka, ${ }^{4} \mathrm{Y}$ Matsuno, ${ }^{2,3} \mathrm{Y}$ Hatanaka, ${ }^{1} \mathrm{H}$ Watari. ${ }^{1}$ Hokkaido University, Department of Obstetrics and Gynecology, Sapporo, Japan; ${ }^{2}$ Hokkaido University Hospital, Center for Development of Advanced Diagnostics, Sapporo, Japan; ${ }^{3}$ Hokkaido University Hospital, Research Division of Genome Companion Diagnostics, Sapporo, Japan; ${ }^{4}$ Hokkaido University Hospital, Department of Surgical Pathology, Sapporo, Japan

\subsection{6/ijgc-2021-ESG0.593}

Introduction/Background* Niraparib, a PARP inhibitor, causes synthetic lethality in tumors with homologous recombination deficiency (HRD) and is now approved for ovarian cancer.
However, the genetic alterations related to HRD rarely occur in ovarian cancers other than high-grade serous carcinoma or endometrioid carcinoma, thus the efficacy of niraparib for rare subtypes of ovarian cancer remains unclear. In this study, we investigated the efficacy of niraparib using Patient-DerivedXenograft (PDX) models with rare subtypes of ovarian cancer and correlated between the efficacy and the expression levels of SLFN11 and ARID1A, a sensitizer of DNA-targeted therapies and a key component of the SWI/SNF complex, respectively.

Methodology We consecutively collected a total of 11 tissue specimens with a preoperative diagnosis of ovarian cancer which was surgically resected in our hospital. These tumors were directly transplanted subcutaneously into NOG mice and PDXs were established. The histologic characteristics were compared between parental tumors and PDX ones. Immunohistochemical (IHC) staining for ARID1A (D2A8U, Cell Signaling Technology) and SLFN11(D-2 sc-515071X, Santa Cruz) and genetic alterations of 160 cancer-related genes including ARID1A (Human Comprehensive Cancer Panel, Qiagen) were performed using the parental tumors. Response to carboplatin (CBDCA) and niraparib was analyzed using the PDX models.

Result(s)* PDXs were established for each one case of carcinosarcoma (CS), adenocarcinoma (Adeno) with neuroendocrine carcinoma (NEC), and clear cell carcinoma (CCC). In histological comparison, PDX tumors generally mimicked the parental tumors. Loss of ARID1A in IHC was found in CCC and CS cases, and genetic alteration of ARID1A was also detected in the CCC case. Positive staining for SLFN11 was found in CS and Adeno with NEC, both of which had TP53 alterations. In the PDX of CS, both CBDCA and niraparib suppressed tumor growth in a dose-dependent manner. In the PDX of Adeno with NEC, CBDCA significantly suppressed tumor growth, while niraparib did not. The efficacy of CBDCA and niraparib is currently under consideration in the PDX of CCC.

Conclusion* The combination of ARID1A and SLFN11 in IHC may be an efficacy biomarker for niraparib in rare subtypes of ovarian cancer. We plan to increase the sample size in the future. 